\title{
Revisión del modelo docente en Proyectos de Ingeniería mediante la aplicación de metodologías innovadoras
}

\section{Review of the teaching model in Engineering Projects through the application of innovative methodologies}

José ANTONIO VÉLEZ Godiño

ORCID: https://orcid.org/0000-0002-3470-9435

Universidad de Sevilla

Departamento de Ingeniería de la

Construcción y Proyectos de Ingeniería

jvelez1@us.es

Fecha de recepción: 13-06-2019

Fecha de aceptación: 28-06-2019

DOI: http://dx.doi.org/10.12795/9788447221912.039

Pp.: 886-908 


\section{Resumen}

En el presente trabajo se describen una serie de innovaciones desarrolladas en el marco de la Red de Formación e Innovación Docente (REFID) del Programa de Formación e Innovación Docente del Profesorado 2019 de la Universidad de Sevilla. El propósito de este trabajo consiste en la descripción de una experiencia real derivada de la aplicación de la metodología basada en los Ciclos de Mejora en el Aula (CIMA) a una asignatura perteneciente a la rama de ingeniería. En primer lugar, se caracterizará el contexto en el que ha tenido lugar la experiencia, procediéndose posteriormente a exponer los detalles correspondientes al diseño, desarrollo y valoración del mencionado proceso de mejora.

Palabras clave: Proyectos, Grado en Ingeniería de la Energía, Docencia Universitaria, Experimentación Docente Universitaria, Taller Conceptual.

\section{Abstract}

This paper describes a series of innovations developed within the framework of the "Red de Formación e Innovación Docente" (REFID) of the 2019 "Programa de Formación e Innovación Docente del Profesorado" of the University of Seville. The purpose of this work is to describe a real experience derived from the application of the methodology based on the Improvement Cycles in Classroom (ICIC) to a subject belonging to the engineering branch. Firstly, the context in which the experience has taken place will be characterized, proceeding later to expose the details corresponding to the design, development and evaluation of the aforementioned improvement process.

Keywords: Projects, Degree in Energy Engineering, University Teaching, University Teaching Experience, Conceptual Workshop. 


\section{Contexto del ciclo de mejora}

La asignatura en la que se desarrolla el Ciclo de Mejora en el Aula (CIMA) descrito corresponde a "Proyectos", asignatura cuatrimestral obligatoria del 3er curso del Grado en Ingeniería de la Energía (GIE), aunque también se imparte en otras titulaciones pertenecientes a la rama de Ingeniería y Arquitectura. La docencia de la mencionada asignatura se articula en 3 bloques (teoría, práctica y prácticas informáticas), abarcando la presente propuesta únicamente la docencia del segundo de ellos, es decir, de la parte práctica. Este bloque se compone de 14 sesiones semanales de 2 horas cada una. Según el plan docente vigente, mediante el bloque práctico:

"Se pretende reproducir el trabajo y la forma de llevar a cabo el desarrollo de un proyecto del ámbito de la ingeniería, con un alcance similar a un anteproyecto. A los alumnos integrados en cada uno de los grupos de trabajo se les asignan tareas, que se van orientando y coordinando con la participación de todos los alumnos del grupo para conseguir los objetivos comunes del proyecto."

De esta forma, la docencia objeto del CIMA consiste en una suerte de juego de rol con un grupo de unos 20 alumnos, cuyo objetivo común es realizar un proyecto básico de ingeniería, el cual cambia en cada edición del curso. Por consiguiente, es crítico resaltar que la docencia afectada no se fundamenta en un temario estático que impartir, sino que mediante esta asignatura se pretende abordar una serie de contenidos con una marcada componente procedimental.

Respecto al perfil de la asignatura objeto de la innovación se caracteriza de forma mayoritaria por un alumnado cuyo principal objetivo es aprobar la asignatura invirtiendo los menores recursos posibles, identificándose 
tradicionalmente pocos casos con verdadero afán de profundización en los contenidos de la asignatura.

El antecedente personal al presente CIMA lo constituye una experiencia de 6 horas de duración desarrollada también para la asignatura "Proyectos" del GIE en el curso 2017-2018 (Vélez, 2018), en el marco del Curso General de Docencia Universitaria (GDU) del Programa de Formación e Innovación Docente del Profesorado (FIDOP) 2018 de la Universidad de Sevilla. Se resumen a continuación las principales conclusiones alcanzadas en aquella ocasión:

- Contenidos: Se reforzó la creencia en la necesidad de priorizar los contenidos procedimentales frente a los conceptuales.

- Metodología: Se identificó la necesidad de ir generando progresivamente actividades del tipo "talleres conceptuales" (Finkel, 2008), que sustituyesen las clásicas sesiones magistrales previamente empleadas con carácter exclusivo.

- Evaluación: Sin lugar a duda, el mayor avance personal se identificó en relación a la evaluación, pasando a concebirse como una herramienta para detectar dificultades en el aprendizaje del alumnado y adaptar los contenidos, no únicamente como un elemento para calificar al alumnado.

A pesar de los logros anteriormente mencionados, el análisis crítico del anterior CIMA puso de manifiesto la necesidad de mejorar en relación a la planificación, ya que determinadas actividades hubieran requerido una mayor dedicación, lo cual no fue viable debido principalmente a los requerimientos temporales del propio curso GDU.

Asimismo, cabe destacar la enriquecedora experiencia derivada del curso sobre mapas de contenidos ofertado por el Programa FIDOP de la Universidad de Sevilla, cuya participación (Octubre-Noviembre 2018) sirvió para 
ampliar los conocimientos en relación a esta útil herramienta docente.

Como conclusión, de cara al presente CIMA se tendrán en cuenta los siguientes principios didácticos derivados de la experiencia anterior:

- Desarrollo de una planificación detallada y dotada de suficientes márgenes de maniobra para a llevar a cabo modificaciones asociadas a las necesidades específicas del alumnado.

- En la medida de lo posible, aplicación de mecánicas de clase basadas en talleres conceptuales, donde tenga cabida el trabajo en grupo en el aula.

- Necesidad de evaluar de forma continua y de realimentar al alumnado en relación a su desempeño, de forma que se le proporcione capacidad de respuesta ante niveles de aprendizaje inadecuados.

\section{Diseño previo}

Respecto al CIMA desarrollado, el cual abarca 18 horas lectivas, cabe destacar que pertenece a la tipología "encadenado", ya que en la práctica se compone de tres CIMA parciales, todos ellos amparados bajo la pregunta clave "¿Cómo se realiza el anteproyecto de una instalación industrial?"

\section{Mapas de contenidos y secuencias de actividades}

Como se ha mencionado con anterioridad, para la elaboración de los mapas de contenidos se han aplicado las nociones derivadas del curso "Los Mapas de Contenidos y Problemas: otra Manera de Diseñar la Enseñanza" (Octubre-Noviembre 2018). De esta forma, se pretende que todos los contenidos abordados estén precedidos de una pregunta que motive la curiosidad del alumnado, a la vez 
que sirva para contextualizar el contenido abordado. En las Figuras 1, 2 y 3 se muestran los mapas de contenidos propuestos para los bloques 1 (¿Cómo se planifica un anteproyecto?), 2 (¿Cómo se caracteriza una instalación industrial?) y 3 (¿Cómo se cuantifica el coste de un proyecto?) respectivamente, marcándose en gris las preguntas clave y en blanco los contenidos desarrollados.

A continuación, se expone la secuencia de actividades correspondiente a cada uno de los tres bloques que articulan el presente CIMA, las cuales están directamente relacionadas con los mapas de contenidos previamente referidos:

- Pregunta clave de la innovación: ¿Cómo se realiza el anteproyecto de una instalación industrial?

- Bloque 1: ¿Cómo se planifica un anteproyecto? (6 horas)

- Actividad 0: Realización del cuestionario inicial (15 minutos).

1. ¿Qué hay que hacer para planificar?

- Actividad 1: Definición del alcance del anteproyecto (25 minutos).

- Actividad 2: Generación de un listado de documentos (55 minutos).

2. ¿Depende la planificación de los recursos disponibles?

- Actividad 3: Definición del organigrama de proyecto (25 minutos).

3. ¿Cómo se ordenan las actividades de un proyecto?

- Actividad 4: Elaboración de un listado de datos de partida requeridos para cada actividad (60 minutos).

- Actividad 5: Identificación de dependencias entre actividades (30 minutos).

- Actividad 6: Asignación de prioridades a las actividades (30 minutos).

4. ¿Cómo se materializa la programación de un proyecto? 
- Actividad 7: Visualización tutorial software de planificación (5 minutos).

- Actividad 8: Realización de la programación del anteproyecto (1 hora 45 minutos).

- Actividad 9: Identificación de la ruta crítica (10 minutos).

5. ¿Cómo se controla la programación?

- Actividad 10: Control semanal de la planificación (15 minutos cada semana).

- Bloque 2: ¿Cómo se caracteriza una instalación industrial? (8 horas)

- Actividad 0: Realización del cuestionario inicial (15 minutos).

1. ¿Cuál es el contexto de un anteproyecto?

- Actividad 1: Sintetizar los objetivos y el alcance del anteproyecto (15 minutos).

2. ¿Qué requisitos FORMALES debe cumplir el anteproyecto?

- Actividad 2: Definición de normas y códigos de diseño aplicables (20 minutos).

- Actividad 3: Definición de procedimientos de trabajo (20 minutos).

- Actividad 4: Definición de plantillas de documentos y de simbología aplicable (20 minutos).

3. ¿Cómo opera la instalación industrial?

- Actividad 5: Caracterización del diagrama de proceso básico (30 minutos).

- Actividad 6: Generación de los balances de masa y energía (40 minutos).

- Actividad 7: Definición de los distintos modos de operación de la instalación (20 minutos).

4. ¿Qué características deberán tener los distintos componentes de la instalación?

- Actividad 8: Caracterización del diagrama tuberías e instrumentación (Piping \& Instrumentation Diagram, P\&ID) (60 minutos). 
- Actividad 9: Dimensionamiento de los equipos críticos (cálculo, especificación y hoja de datos) (90 minutos).

- Actividad 10: Caracterización del resto de componentes (especificación) (30 minutos).

5. ¿Dónde se debe ubicar cada equipo?

- Actividad 11: Plano de implantación general (45 minutos).

- Actividad 12: Planos de ruteados (tuberías y cableado) (15 minutos).

- ¿Qué componentes son necesarios?

- Actividad 13: Revisión del P\&ID (15 minutos).

- Actividad 14: Generación de listados de componentes (tuberías, cables e instrumentos) (45 minutos).

Bloque 3: ¿Cómo se cuantifica el coste de un proyecto? (4 horas)

- Actividad 0: Realización del cuestionario inicial (15 minutos).

1. ¿Qué elementos componen un presupuesto?

a) Actividad 1: Diferenciar entre medición y presupuesto (15 minutos).

2. ¿De dónde procede la información necesaria para elaborar la medición?

- Actividad 2: Caracterización de las unidades de obra principales (20 minutos).

- Actividad 3: Caracterización de las unidades de obra secundarias (10 minutos).

3. ¿De dónde procede la información necesaria para cuantificar el coste de una unidad de obra?

- Actividad 4: Procedimiento con datos disponibles (20 minutos).

- Actividad 5: Procedimiento sin datos disponibles (escalado y actualización de costes) (40 minutos).

4. ¿Cómo se mide la rentabilidad de un proyecto?

- Actividad 6: Definición de indicadores de rentabilidad (30 minutos). 
5. ¿Cómo se calculan los distintos indicadores de rentabilidad?

- Actividad 7: Generación de una hoja de cálculo de indicadores de rentabilidad (60 minutos).

6. ¿Qué datos de partida son necesarios para calcular los distintos indicadores de rentabilidad?

- Actividad 8: Generación de una plantilla de datos de partida (15 minutos).

7. ¿Qué peculiaridades posee el cálculo de los distintos indicadores de rentabilidad?

- Actividad 9: Análisis de los detalles de cálculo del "coste anualizado de la electricidad" (5 minutos).

- Actividad 10: Determinación de la duración óptima de un préstamo (5 minutos).

- Actividad 11: Análisis del cálculo de los impuestos correspondientes (5 minutos).

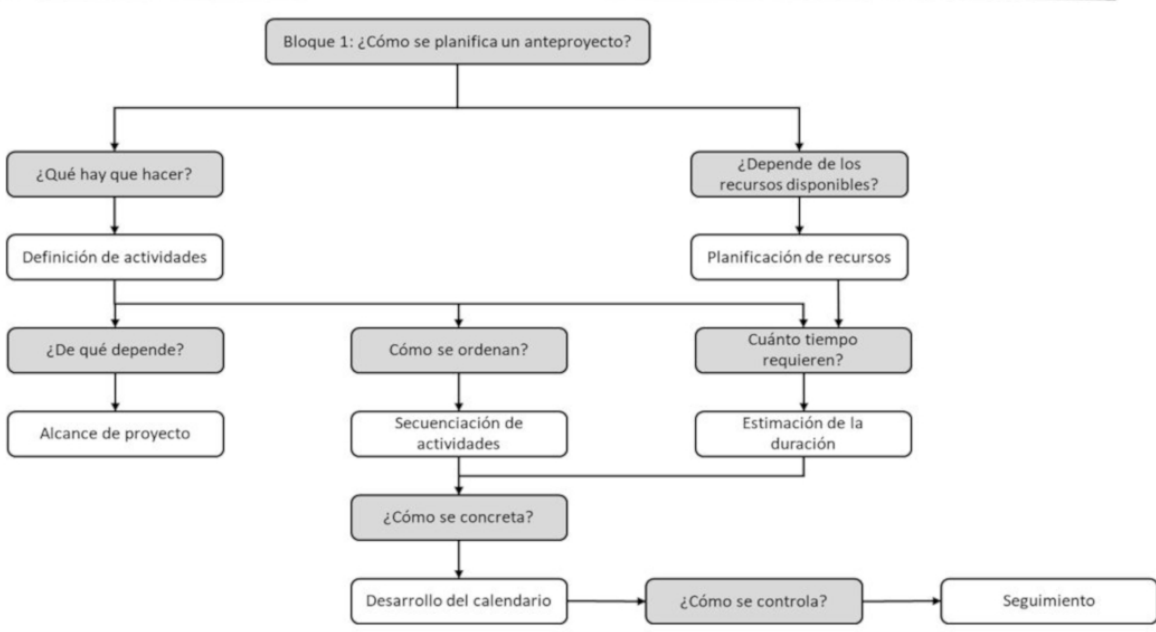

Figura 1. Mapa de contenidos del bloque 1 (¿Cómo se planifica un anteproyecto?)

Jornadas de Formación e Innovación Docente del Profesorado I № 2 (2019) Esta obra se distribuye con la licencia Creative Commons 


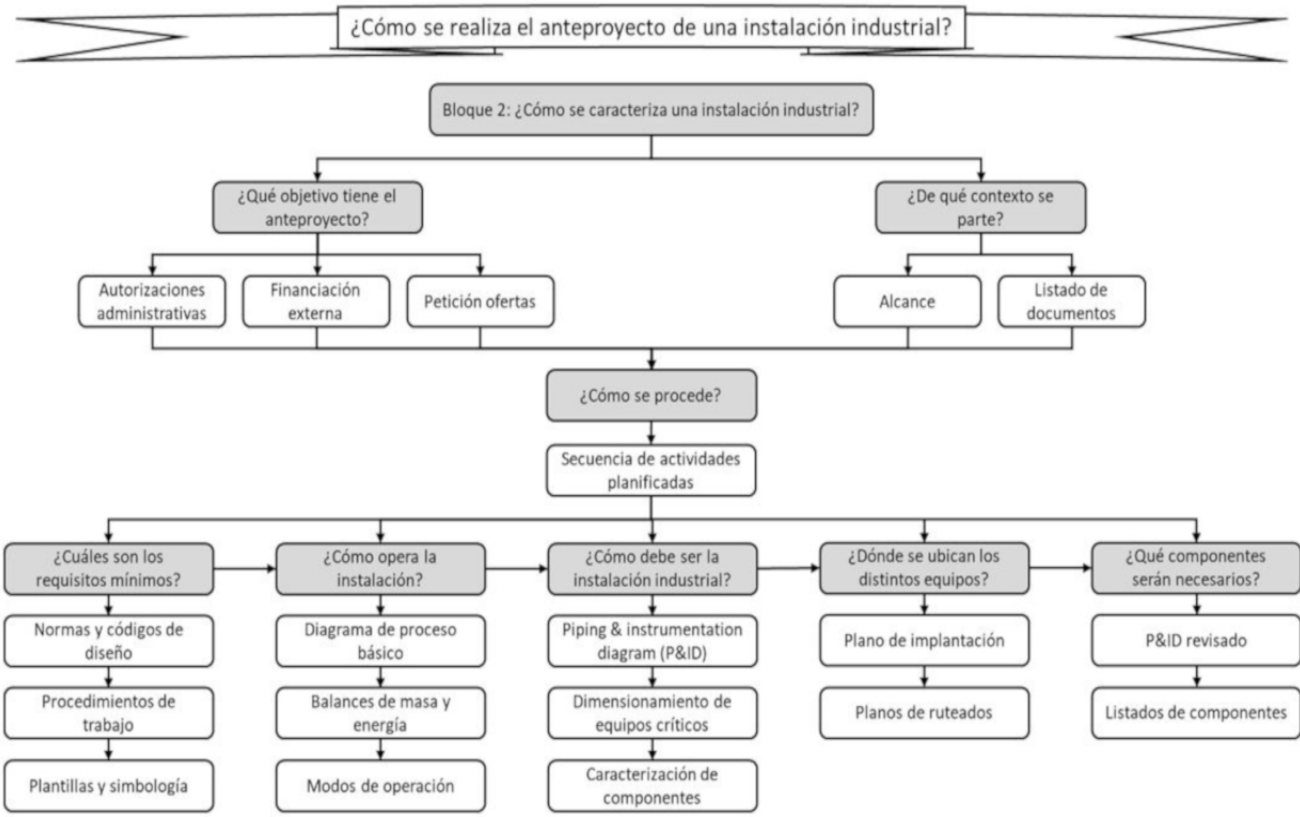

Figura 2. Mapa de contenidos del bloque 2 (¿Cómo se caracteriza una instalación industrial?)

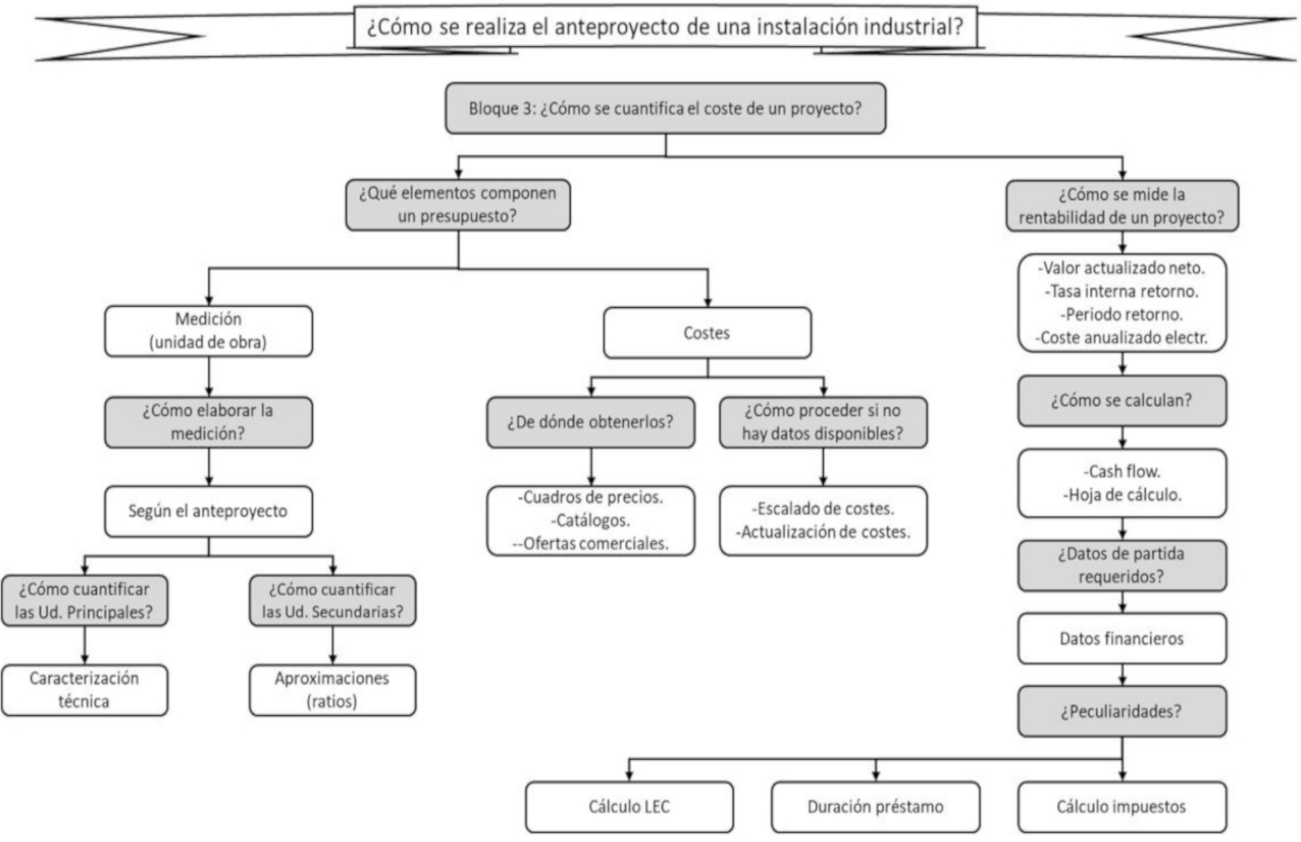

Figura 3. Mapa de contenidos del bloque 3 (¿Cómo se cuantifica el coste de un proyecto?)

Jornadas de Formación e Innovación Docente del Profesorado | № 2 (2019)

Esta obra se distribuye con la licencia Creative Commons

Reconocimiento-NoComercial-SinObraDerivada

4.0

Internacional (CC BY-NC-ND 4.0.) 


\section{Modelo metodológico}

Antes de proceder al análisis del modelo metodológico empleado para el desarrollo de las innovaciones descritas en el presente documento, conviene indicar que el autor del mismo posee un perfil que podría englobarse en el "modelo tecnológico de profesorado" derivado de la crítica racionalista al modelo tradicional y caracterizado por estar centrado principalmente en los contenidos impartidos (Porlán, 2017).

Al igual que en relación al CIMA realizado previamente, el modelo metodológico ideal debería poseer una naturaleza exclusivamente práctica (ver Figura 4). Es decir, el modelo debería reproducir la práctica habitual en un proyecto real de ingeniería. Según este enfoque, el desarrollo de la asignatura debería estar completamente gestionado por los alumnos, pasando el profesorado a actuar como un cliente final, no entrando a valorar el desarrollo de las tareas sino simplemente en los resultados finales obtenidos. La principal ventaja de este enfoque radicaría en una experiencia de aprendizaje más sólida, al ser el alumnado el único responsable de su aprendizaje. Respecto a las dificultades que entraña este planteamiento cabe destacar:

- Incapacidad del alumnado para aplicar los conocimientos teóricos previamente adquiridos, así como falta de motivación para profundizar en las tareas desarrolladas.

- Falta de confianza por parte del profesor, debido al alto riesgo de bloqueo del proyecto derivado tanto de la menor velocidad de avance como de la limitante duración de la asignatura (cuatrimestral).

Debido a las limitaciones anteriores, el modelo metodológico ideal previamente expuesto requiere ser modificado, generándose el modelo metodológico posible mostrado en la Figura 5, el cual posee tres modificaciones respecto al planteamiento inicial: 
- En base a la experiencia previa y al material bibliográfico analizado, se considera imprescindible la intervención del profesorado en al inicio de cada bloque, con el objeto de plantear las preguntas clave requeridas para generar el interés del alumnado en relación a los contenidos que se pretende abordar.

- Tras una primera fase de trabajo autónomo por parte del alumnado, se propone llevar a cabo una revisión colaborativa de la misma, en la que participe de forma activa, no sólo el profesorado, sino también el resto del alumnado, aunque no hayan participado activamente en el desarrollo de la tarea revisada. Los objetivos de esta fase son tres (Bain, 2007):

- Al exponer el trabajo realizado el alumnado logra una mayor comprensión del contenido.

- La participación de alumnos no vinculados al desarrollo de la tarea implica un punto de vista externo, el cual puede llegar a ser enriquecedor.

- Por último, la participación del profesorado previene la aparición de errores graves, a la vez que posibilita la evaluación continua del desempeño del alumnado.

- La tercera modificación consiste en que el alumnado lleve a cabo la reformulación de las ideas iniciales, como resultado de las principales conclusiones alcanzadas durante la revisión colaborativa previa. De esta forma se pretende la revisión de los modelos mentales previos y su actualización por esquemas más complejos.
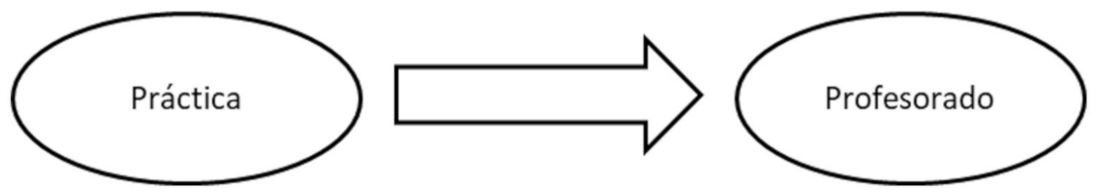

Figura 4: Modelo metodológico ideal

Jornadas de Formación e Innovación Docente del Profesorado | № 2 (2019) Esta obra se distribuye con la licencia Creative Commons Reconocimiento-NoComercial-SinObraDerivada 4.0 Internacional (CC BY-NC-ND 4.0.) 


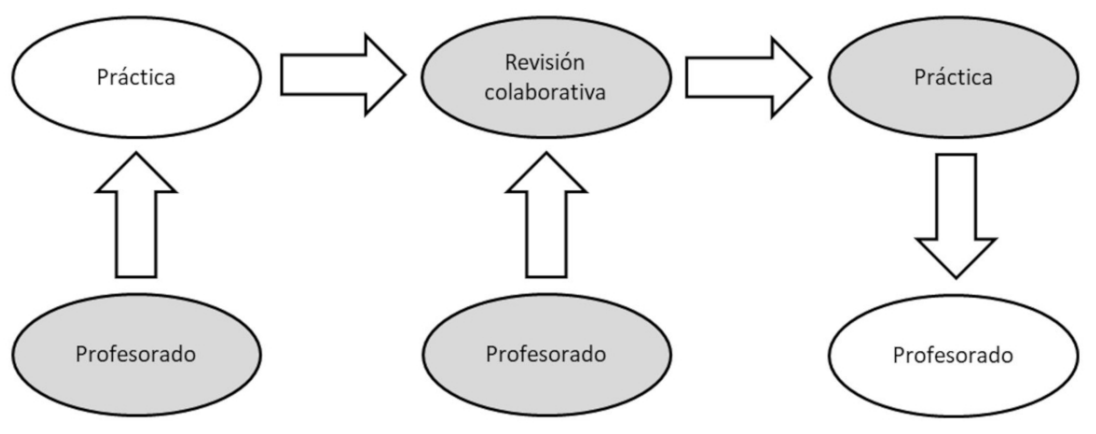

Figura 5: Modelo metodológico posible (en gris, las modificaciones respecto a la Figura 4)

\section{Cuestionarios de exploración}

A continuación, se expone el cuestionario de exploración correspondiente a cada uno de los tres bloques que articulan el presente CIMA. Puede observarse que las preguntas han sido modificadas ligeramente respecto a las recogidas en los mapas de contenidos y en las secuencias de actividades anteriormente expuestas. La finalidad de esta modificación no es otra que lograr que las preguntas resulten abiertas y estimulantes.

- Pregunta clave de la innovación: ¿Cómo se realiza el anteproyecto de una instalación industrial?

- Bloque 1: ¿Cómo se planifica un anteproyecto?

1. Para iniciar la programación del proyecto, ¿cuál cree que es la primera acción que debe realizarse?

2. ¿De qué factores depende la acción mencionada en su respuesta anterior?

3. ¿Cómo ordenaría las actividades requeridas para la realización de un proyecto?

4. ¿Es necesario estimar a priori la duración de cada actividad de un proyecto?

5. ¿Cómo se materializa la programación de un proyecto?

6. ¿Cómo se controla la programación de un proyecto? 
- Bloque 2: ¿Cómo se caracteriza una instalación industrial?

1. ¿Cuáles son los objetivos primordiales de un anteproyecto?

2. ¿Cree que es necesario organizar la secuencia de tareas que componen el anteproyecto? Justifique su respuesta.

3. ¿Podría identificar requerimientos aplicables a todas las disciplinas involucradas en un anteproyecto?

4. ¿Qué tareas considera que deben ser las iniciales en relación a la caracterización técnica de una instalación industrial?

5. En relación a la pregunta anterior, ¿qué tareas considera que deben ser el núcleo de la caracterización técnica de una instalación industrial?

6. "La ubicación de los distintos elementos de la instalación no influye en el anteproyecto porque éste está compuesto por información preliminar". ¿Está de acuerdo con esta afirmación? Justifique su respuesta.

7. De cara a la realización de un presupuesto, ¿con qué tipo de actividades cree que debería acabar un anteproyecto?

- Bloque 3: ¿Cómo se cuantifica el coste de un proyecto?

1. ¿Qué elementos básicos componen un presupuesto?

2. ¿De dónde procede la información necesaria para elaborar una medición?

3. ¿Poseen la misma relevancia todas las unidades de obra que componen una medición?

4. ¿De dónde procede la información necesaria para cuantificar el coste de una unidad de obra?

5. ¿Existen mecanismo para cuantificar el coste de una unidad de obra cuyo precio unitario es desconocido a priori?

6. ¿Cómo se mide la rentabilidad de un proyecto?

7. ¿De qué herramientas se dispone para calcular los distintos indicadores de rentabilidad?

8. ¿Qué datos de partida son necesarios para calcular los distintos indicadores de rentabilidad? 


\section{Aplicación del ciclo de mejora}

\section{Desarrollo de las sesiones}

Conviene aclarar en primer lugar que el ambiente en el que se desarrollaron las sesiones que constituyeron el CIMA descrito en este trabajo está condicionado por el pequeño tamaño del grupo de trabajo, lo que permite que el profesorado conozca a cada miembro del alumnado, lo cual, a juicio del autor, representa una clara ventaja al facilitar la adaptación a las dificultades típicas de la actividad docente. Otro elemento que conviene resaltar deriva del desconocimiento por parte del alumnado de su participación en el CIMA aquí descrito. La decisión por parte del autor de no informar al alumnado sobre este extremo deriva de la intención de evitar posibles resistencias injustificadas por parte del alumnado al cambio, independientemente de que éste suponga una ventaja o un inconveniente. Para finalizar con la caracterización del clima de trabajo experimentado durante el desarrollo del CIMA podría concluirse que la atmósfera ha sido positiva con carácter general, aunque no tan participativa ni motivada como se esperaba por parte del autor.

Respecto a la valoración de la actuación propia, el autor destaca que ésta se vio condicionada desde el primer momento por la previamente mencionada carencia de motivación generalizada entre el alumnado, la cual supuso la necesidad replantear ciertas decisiones durante el desarrollo del bloque 1 del CIMA. Un análisis crítico por parte del autor achacó la falta de motivación, en primer lugar, al anteriormente mencionado perfil dominante en el seno del alumnado, el cual se caracteriza por minimizar los esfuerzos requeridos para lograr un aprobado, adoleciendo de una verdadera vocación de aprendizaje profundo y duradero. Sin embargo, gracias al feedback continuamente recibido durante el desarrollo del CIMA como consecuencia de la evaluación continua, también se pudo poner de 
manifiesto que la falta de motivación inicial del alumnado derivaba, al menos parcialmente, de las dificultades experimentadas al enfrentarse a tareas con múltiples grados de libertad, como ocurre en los talleres conceptuales, a diferencia de las tareas, típicamente repetitivas, que están habituados a realizar en el marco del frecuentemente empleado modelo docente consistente casi exclusivamente en la impartición de sesiones magistrales. Como medida encaminada a mitigar el impacto de la mencionada falta de motivación del alumnado se decidió reformular el diseño inicialmente previsto. A pesar de que en un principio los mapas de contenidos desarrollados no eran mostrados al alumnado, tras las dificultades iniciales se consideró adecuado entregar al alumnado los mapas de contenidos, con la intención de proporcionarle un soporte que le ayudase a generar una visión de conjunto del problema a resolver y, de esta forma, realizar una transición suave a la realización de talleres conceptuales.

A continuación, se caracteriza el desarrollo de actividades de cada uno de los bloques que componen el CIMA objeto del presente trabajo:

- Bloque 1: Como se ha adelantado, durante el desarrollo del primer bloque tuvieron lugar las principales dificultades detectadas durante el desarrollo del CIMA. La principal consecuencia de estas dificultades fue la necesidad de reformular el modelo docente planteado, lo que conllevó un mayor consumo de tiempo del estimado inicialmente. Este mayor consumo de tiempo tuvo consecuencias adversas que serán expuestas en el análisis del bloque 3.

- Bloque 2: Tras los ajustes llevados a cabo en el primer bloque, el bloque 2 se desarrolló sin ninguna incidencia. También contribuyó al correcto desarrollo de este bloque que las actividades planteadas, si bien resultaban abiertas, presentaban cierta similitud a las tareas de perfil técnico que el alumnado está más acostumbrado a desempeñar. En relación a este bloque cabe destacar la puesta en práctica por parte del alumnado de un contenido procedimental 
tan básico como la optimización de los procesos de toma de decisiones.

- Bloque 3: Debido al mayor consumo de tiempo referido en relación al bloque 1, el desarrollo del bloque 3 se vio penalizado, siendo necesario descartar las actividades desde la no 4 hasta la no 7 del bloque 3. Si bien mediante estas actividades se pretendía proporcionar una visión práctica, no resultó una renuncia crítica, ya que los contenidos descartados se abordan tanto en la parte teórica de la asignatura como en otras asignaturas de la rama de economía. En relación a este bloque es preciso destacar que, a pesar del recorte en los contenidos abordados, el alumnado se centró satisfactoriamente en un contenido de tipo procedimental que desconocían hasta el momento: la estimación de costes en el ámbito de la ingeniería.

Según el relato previamente expuesto en relación del desarrollo del presente CIMA cabría concluir que la principal dificultad consistió en la falta de motivación inicial del alumnado, la cual se achaca en parte al cambio de paradigma asociado al desarrollo de los talleres conceptuales, en los cuales la solución no está tan definida como en la mayoría de los problemas resueltos durante la estancia en la universidad. Como se expuso con anterioridad, para solventar esta dificultad se recurrió a los mapas de contenido, con el objeto de proporcionar una visión global del problema planteado.

A pesar de esto, el desarrollo del presente CIMA también ha permitido poner de manifiesto notables avances en relación al tratamiento de contenidos procedimentales, los cuales, a juicio del autor, reciben típicamente una menor dedicación que los contenidos conceptuales. En relación con este aspecto cabe destacar las notables mejoras en relación al ejercicio de la capacidad de toma de decisiones, el cual desempeñará un papel crucial en el posterior ejercicio profesional del alumnado. 


\section{Evaluación del aprendizaje}

Para proceder a la evaluación de la experiencia de aprendizaje derivada del CIMA descrito en este trabajo se recurrió a la elaboración de escaleras de aprendizaje, las cuales a su vez se confeccionaron a partir de los resultados derivados de los cuestionarios de exploración realizados antes y después del desarrollo de cada uno de los tres bloques en que se articuló el CIMA. Asimismo, conviene destacar que metodología de análisis de resultados se basó en agrupar, ordenar, analizar y, por último, establecer conclusiones (Giné, 2000). Antes de proceder al análisis de los resultados conviene aclarar que, debido al recorte de contenidos efectuado en el bloque 3 , no se han tenido en cuenta las respuestas a las preguntas 6, 7 y 8 del cuestionario correspondiente.

El resultado obtenido se basa en la clasificación de las respuestas en cuatro niveles de conocimiento, siendo el nivel 1 el más superficial, mientras que el nivel 4 constituye un nivel superior de aprendizaje. En las Figuras 6, 7 y 8 se muestran los resultados promedio de cada uno de los bloques, considerando el mismo peso porcentual para todas las preguntas. En la totalidad de los cuestionarios iniciales el alumnado se dividió entre los niveles 1 y 2 , mientras que en los cuestionarios finales los niveles dominantes eran el 2 y el 3. Mención especial requiere el resultado del cuestionario final del bloque 3, donde llama la atención el porcentaje de alumnado que alcanzó el nivel 4.

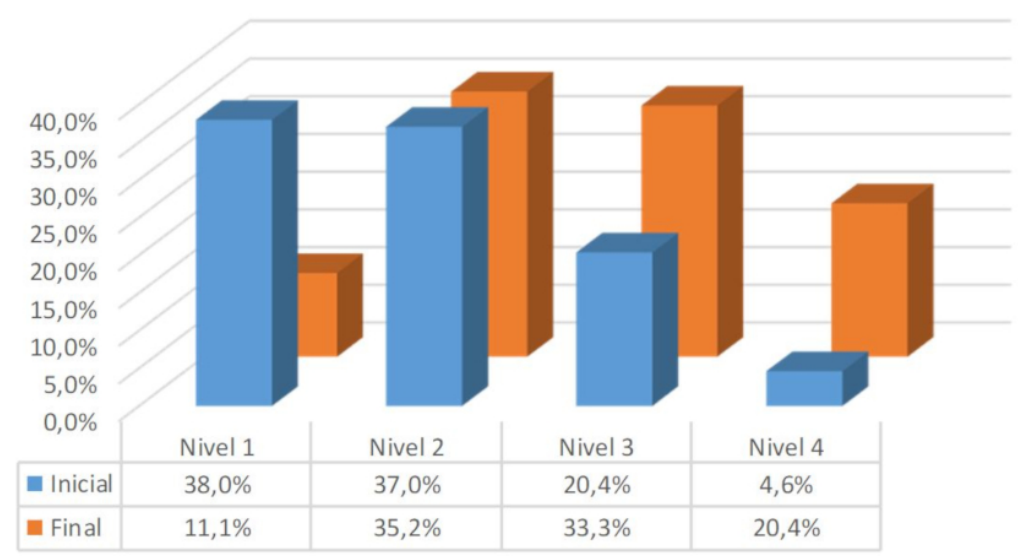

Figura 6: Resultado promedio cuestionarios bloque 1

Jornadas de Formación e Innovación Docente del Profesorado | № 2 (2019) Esta obra se distribuye con la licencia Creative Commons Reconocimiento-NoComercial-SinObraDerivada Internacional (CC BY-NC-ND 4.0.) 


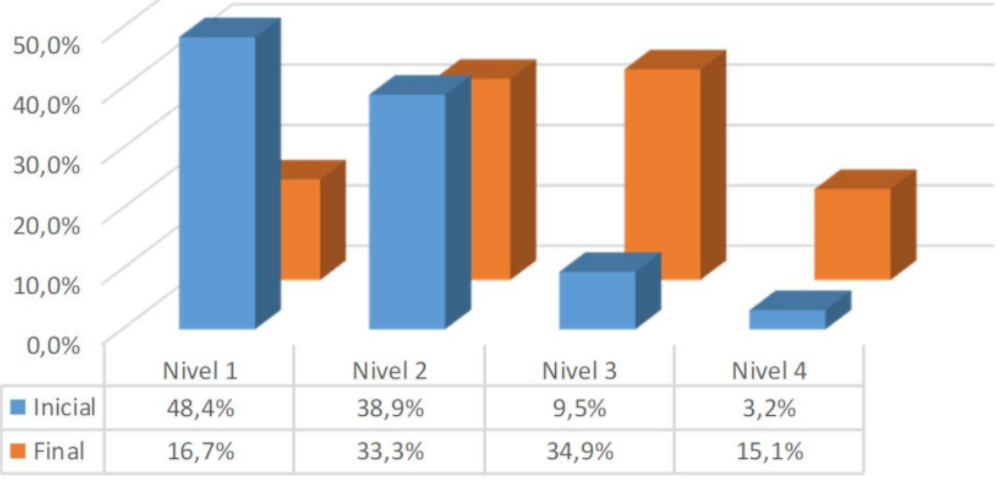

Figura 7: Resultado promedio cuestionarios bloque 2

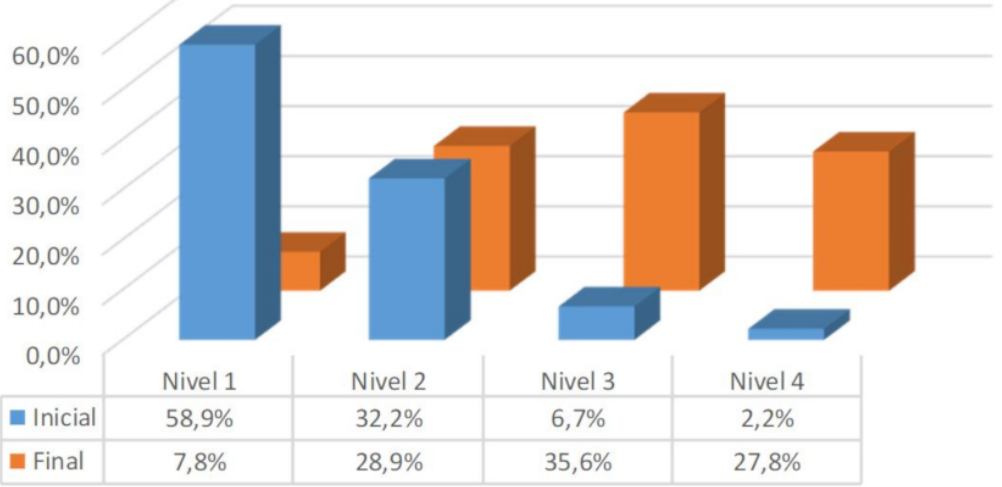

Figura 8: Resultado promedio cuestionarios bloque 3

Además de los mencionados cuestionarios de exploración conviene destacar que, dado que la evaluación continua constituye uno de los principios didácticos de este CIMA, se han empleado medidas complementarias para la evaluación, como son:

- Seguimiento semanal individualizado, proporcionando al alumnado un feedback rápido mediante comentarios derivados de la corrección de las tareas

Jornadas de Formación e Innovación Docente del Profesorado | № 2 (2019) Esta obra se distribuye con la licencia Creative Commons Reconocimiento-NoComercial-SinObraDerivada 
realizadas. Cabe destacar que, gracias a esta práctica, se pudo advertir con suficiente antelación a uno de los equipos de trabajo que, de no realizar cambios en su desempeño, la calificación final no sería positiva.

- Fichas de desempeño semanales, en las que cada equipo definía a qué tareas del proyecto se había dedicado cada integrante durante la semana previa a la sesión formativa. Finalmente, estas fichas demostraron poseer una escasa utilidad.

- Incluir en la evaluación final un apartado dedicado a evaluación por pares "adaptada”, mediante la cual, el alumnado no pone notas a sus compañeros de trabajo, sino que establece una clasificación ordenada dentro de su equipo de trabajo atendiendo a diferentes criterios relacionados con las competencias establecidas en el programa de la asignatura.

\section{Evaluación del CIMA}

La evaluación del CIMA desarrollado se ha llevado a cabo mediante la combinación de los siguientes elementos:

- Observación directa por parte del profesorado, especialmente en la fase designada como "revisión colaborativa" del modelo metodológico posible.

- Redacción de informes de trabajo semanales por parte del alumnado, aunque como se ha expuesto con anterioridad, esta herramienta degeneró en un elemento polémico entre el alumnado, además de cumplimentarse con falta de rigor.

- Aunque inicialmente se valoró la elaboración de un diario del profesor, finalmente se procedió al registro únicamente de notas de campo como herramienta de evaluación por parte del profesorado. De esta forma se procedió a tomar nota de palabras clave durante la clase y que éstas actuasen como anclajes que permitían al profesorado una posterior evaluación. 
- Encuesta de opinión al final del CIMA, otorgando al alumnado la posibilidad de valorar, rechazar y proponer.

Entre los aspectos que conviene mantener cabe destacar el papel desempeñado por los mapas de contenidos, especialmente tras haber sido finalmente entregados al alumnado. Entre las ventajas de esta herramienta, además de facilitar la planificación de la práctica docente, el alumnado resalta su gran utilidad para estructurar contenidos. Otro aspecto a mantener debido a los buenos resultados obtenidos consiste en la evaluación continua, la cual posibilita informar al alumnado semanalmente sobre los resultados obtenidos.

En el área de las necesidades de cambios, además del previamente mencionado abandono de las fichas de desempeño semanal, se identifica la necesidad de realizar una transición suave al modelo de actividad basado en el taller conceptual. Esta necesidad deriva del cambio de paradigma que representa este tipo de actividad en comparación con las habitualmente desempeñadas por el alumnado. Como parte de esta transición suave se identifica la necesidad de un mayor guiado por parte del profesorado en las etapas iniciales del proceso, el cual se debe ir reduciendo progresivamente.

Para concluir, se procede a la revisión de los principios didácticos inicialmente planteados:

- Se procede a ratificar la concepción inicial en relación a los contenidos (Vélez, 2018), la cual se basa en la relevancia, en el marco de la asignatura analizada, de los de tipo procedimental en detrimento de los contenidos de tipo conceptual.

- Respecto a la metodología adoptada, a pesar de las grandes expectativas depositadas tras la primera experiencia personal al respecto, los talleres conceptuales se han revelado como una herramienta no 
sólo compleja por sus propias características, sino que además presenta ciertas dificultades adicionales para el alumnado por el hecho de diferir de la práctica habitual en el ámbito universitario. Por el contrario, los resultados derivados del uso de mapas de contenidos han superado las expectativas iniciales, ayudando a mitigar las dificultades previamente referidas. Se considera que, a pesar de las dificultades referidas en relación a la metodología, el mayor avance personal derivado del presente CIMA se ha producido en esta área.

- Finalmente, en relación a la evaluación se ratifica la visión previa al desarrollo del CIMA, recalcando la necesidad de un proceso de evaluación continua que permita la identificación de dificultades por parte del alumnado y la adaptación de los contenidos.

Jornadas de Formación e Innovación Docente del Profesorado | № 2 (2019) Esta obra se distribuye con la licencia Creative Commons 


\section{Referencias bibliográficas}

BAIN, K. (2007). Lo que hacen los mejores profesores universitarios. Valencia: Universitat de Valencia.

FINKEL, D. (2008). Dar clase con la boca cerrada. Valencia: Universitat de Valencia.

GINE, N. y PARCERISA, A. (2000). Evaluación en la educación secundaria. Elementos para la reflexión y recursos para la práctica. Barcelona: Graó.

PORLÂN, R. (2017). ENSEÑANZA UNIVÉRSITARIA Cómo mejorarla. Madrid: Morata.

VÉLEZ GODIÑO, J. A. (2018). Ciclo de mejora en la asignatura Proyectos de la rama de Ingeniería. Jornadas de Formación e Innovación Docente del Profesorado, (1), 832-849. 